\title{
Inpatient boarding in emergency departments: impact on patient delays and system capacity
}

\author{
Raïsa Carmen ${ }^{\mathrm{a}, *}$, Inneke Van Nieuwenhuyse ${ }^{\mathrm{a}, \mathrm{b}}$, Benny Van Houdt ${ }^{\mathrm{c}}$ \\ ${ }^{a}$ Research Center for Operations Management, Department of Decision Sciences and Information Management, KU \\ Leuven, Naamsestraat 69, 3000 Leuven, Belgium \\ ${ }^{b}$ Research Group Logistics, Hasselt University, Agoralaan Building D, 3590 Diepenbeek, Belgium \\ ${ }^{c}$ Department of Mathematics and Computer Science, University of Antwerp, Belgium
}

\begin{abstract}
This study seeks insights into the impact of inpatient boarding on emergency department (ED) congestion and capacity. To do so, we model the ED as a semi-open queueing network (SOQN) with limited resources (physicians and beds) and discontinuous patient service. We present a Markovmodulated fluid queue approach to efficiently calculate service levels, and show that boarding may cause the (expensive) physician resources to be starved, especially when the bed utilization is high. While the expected number of boarding patients has a primary impact on performance, we show that there is a secondary impact stemming from the expected boarding time and the boarding probability. Boarding reduction policies perform better if they focus on reducing expected boarding time instead of the decreasing probability of boarding. Our analysis and insights are applicable also to other SOQN settings where entities require more than one resource simultaneously (e.g., intensive care units, manufacturing systems, warehousing and transportation systems).
\end{abstract}

Keywords: OR in health services, Markov-modulated fluid queue, emergency department, inpatient boarding

\section{Introduction}

Patients often need to wait in the emergency department (ED) to be admitted to the inpatient ward (IW), a step referred to in the medical literature as inpatient boarding, access block, or bed block (Pines et al., 2011). Inpatient boarding represents an important cause of ED crowding (Pines et al., 2011), and occurs because of different reasons (e.g., poor synchronization between the ED and an IW; lack of staff, equipment, and beds in the IW; see Armony et al., 2015; Shi et al., 2015). The consequences for patients are severe: boarding has been associated with patients 'leaving without being seen' (LWBS), poorer patient outcomes, and higher mortality rates (Bernstein et al., 2009, Trzeciak and Rivers, 2003). Despite the practical relevance of this problem, research on this topic has been relatively limited (Saghafian et al., 2015).

\footnotetext{
* Corresponding author

Email addresses: raisa.carmen@kuleuven. be (Raïsa Carmen), inneke.vannieuwenhuyse@uhasselt. be (Inneke Van Nieuwenhuyse), benny.vanhoudt@uantwerpen. be (Benny Van Houdt)
} 
This article presents a generic modelling approach to assess the impact of inpatient boarding on ED congestion and capacity (i.e., maximum throughput that the ED can achieve). Our approach models the ED as a semi-open queueing network (SOQN) with limited resources (physicians and beds) and discontinuous patient service (i.e., patients may need multiple visits with a physician before they can be discharged; see also Yom-Tov and Mandelbaum, 2014). In a SOQN, or open queueing network with population constraints (Buitenhek et al., 2000; Dallery, 1990), exceeding the population constraint means that newly arrived entities must wait in an external queue (Jia and Heragu, 2009). In our study, entities are patients, who might have to wait to obtain an ED bed (the length of this wait defines their external waiting time). The internal ED operations are modelled by a queueing network: patients may have to wait for a physician at each treatment step (internal or physician waiting time), and after treatment, they may have to wait for admission to the IW (i.e., inpatient boarding). We model the resulting network as a Markov-modulated fluid queue (MMFQ) and solve it numerically and exactly. As we will show, this methodology enables us to efficiently calculate service levels (i.e., probability that the waiting time does not exceed an arbitrary threshold) for both queues. Our use of the MMFQ approach is novel; most prior literature uses asymptotic analyses or a quasi-birth-death (QBD) approach. Yet, obtaining service levels from a QBD process is quite involved; while specific approaches exist (see Sengupta, 1989, 1990), the MMFQ approach presented here is far more efficient in this respect (see Section 3.2 for further details). Our results show that boarding affects both congestion (internal and external) and ED capacity. It increases the probability that the (expensive) physician resources are starved, especially when the ED has high bed utilization. While the expected number of boarding patients has a primary impact on performance, we show that there is a secondary effect stemming from the expected boarding time and the boarding probability; consequently, boarding reduction policies that focus on reducing expected boarding time, such as pushing boarding patients out of the ED towards IWs (often waiting in hallways) when the number of boarding patients in the ED reaches a threshold (see Villa-Roel et al., 2012) perform better than those focusing on decreasing probability of inpatient boarding by providing a limited number of beds to accommodate boarding patients (such as acute admission units; see Kolb et al. 2008). Our approach and insights are also applicable to other settings where entities that have finished processing may temporarily block resources, causing other entities to wait (and, possibly, other resources to starve). Essentially, any setting that corresponds to a SOQN may exhibit such behavior. Examples abound in healthcare (for instance, intensive care units, post-anesthesia care units and internal wards), manufacturing systems (e.g., CONWIP systems, see Heragu and Srinivasan, 2011) and warehousing and transportation systems (Dhingra et al., 2018; Roy et al., 2012).

\section{Literature Review}

Although prior studies in computer science, manufacturing systems, and warehousing apply SOQNs extensively to model networks, their uses in healthcare settings have been relatively limited (cf., Yom-Tov, 2010); we refer to Roy (2016) for an overview of applications of and solution methods 


\begin{tabular}{ll}
\hline Notation & Description \\
\hline $\mathbf{1}_{\boldsymbol{i}}$ & A column vector of ones of size $1 \times i$ \\
$\mathbf{0}_{\boldsymbol{i}}$ & A column vector of zeros of size $1 \times i$ \\
$\boldsymbol{I}_{\boldsymbol{i}}$ & An identity matrix of size $i \times i$ \\
$\mathbf{0}_{(\boldsymbol{i} \times \boldsymbol{j})}$ & A zero matrix of size $(i \times j)$ \\
$(a \vee b)$ & Yields the maximum of two real numbers $a, b \in \mathbb{R}$ \\
$(a \wedge b)$ & Yields the minimum of two real numbers $a, b \in \mathbb{R}$ \\
\hline
\end{tabular}

Table 1: Overview of notation

for SOQNs. SOQNs do not have product-form solutions but can be solved numerically using matrixgeometric methods; for complex systems (e.g., many stations, multiclass networks, general arrival or service processes), approximations have been developed. We model the ED as a single-class SOQN with exponential interarrival and service times, and solve it numerically and exactly using an MMFQ approach. This approach can be extended to model general phase-type interarrival and service times, at the expense of longer computation times.

This article relies on and contributes to the extensive literature stream that analyzes patient flow in the ED using queueing theory (see, e.g., Green, 2006; Izady and Worthington, 2012; Saghafian et al., 2014). That is, we regard the treatment process as discontinuous (see also Campello et al., 2016; Huang et al., 2015; Liu and Whitt, 2012; Yom-Tov and Mandelbaum, 2014), because patients may return to the physician for several treatment steps. Yom-Tov and Mandelbaum (2014) model a similar type of service using the Erlang-R queueing model, where $\mathrm{R}$ stands for reentrant customers. Liu and Whitt (2012) let the treatment time and feedback probability depend on the visit number. Queueing models with reentrant patients also have been applied for nurse staffing in IWs de Véricourt and Jennings, 2011; Yankovic and Green, 2011).

Somewhat surprisingly, most ED patient flow models neglect the boarding aspect (cf., Wang, 2013). Most articles that study boarding take the perspective of the IW: see, e.g., Shi et al. (2015) who investigate the impact of IW discharge policies on the time-of-day boarding times in the ED, and Yankovic and Green (2011) who examine the impact of the number of IW beds and nurses on patient boarding probabilities (without examining the impact on ED performance).

\section{Model Description and Methodology}

Table 1 summarizes the notation we use throughout this article.

The ED queueing network is shown in Figure 1 1 . Patients arrive according to a homogeneous Poisson process with rate $\lambda$. The ED disposes of $N$ beds and $s$ physicians, modeled as a SOQN where patients first must obtain a bed before they can undergo treatment by a physician. The wait for bed queue is referred to as the external queue; the physician queue is referred to as the internal queue. Physician treatment times are exponentially distributed with mean $1 / \mu$; after treatment, patients undergo an additional test phase (exponentially distributed with mean $1 / \delta$ ) before returning to the physician with probability $p$. The test phase can represent any treatment that does not involve the physician, such as blood sampling, lab tests, or medical imaging. After physician treatment, 
patients start boarding with probability $(1-p) p_{b}$ or leave with probability $(1-p)\left(1-p_{b}\right)$. The boarding time is exponentially distributed with mean $1 / \beta$.

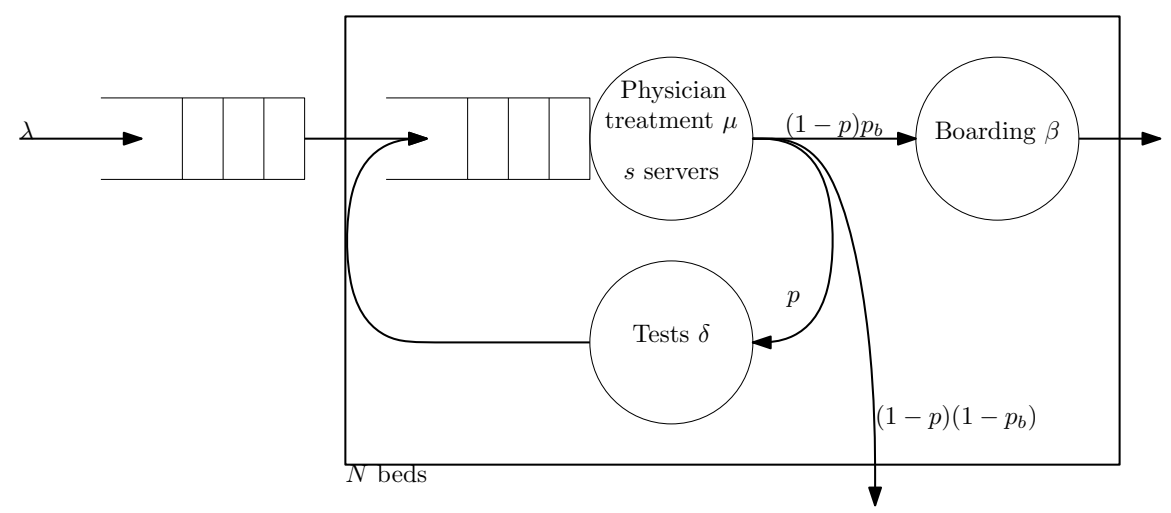

Figure 1: ED queueing network

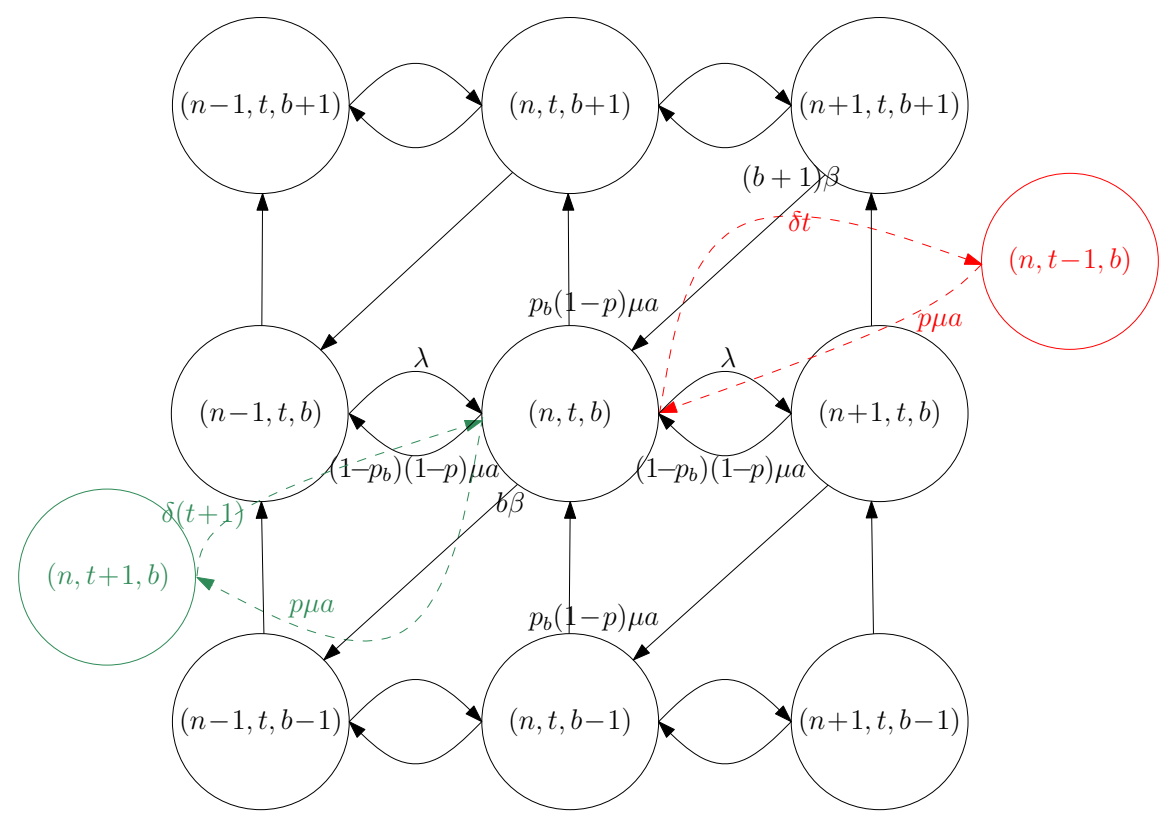

Figure 2: State-transition diagram of the network

The state of the network can be represented by the three-tuple $(n, t, b)$, where $n \in\{0,1, \ldots, \infty\}$ is the total number of patients in the system (including the external queue); $t \in\{0,1, \ldots, N\}$ is the number of patients in the test phase; and $b \in\{0,1, \ldots, N\}$ is the number of boarding patients. These states are ordered lexicographically in all transition matrices. The number of patients in the external queue is $(0 \vee n-N)$, and the number of patients receiving physician treatment (excluding the physician queue) is $a=(((n \wedge N)-t-b) \wedge s)$. Figure 2 shows the state-transition diagram for any $n \in\{1, \ldots, \infty\}$ and $t, b \in\{1, \ldots, N-1 \mid t+b \leq N\}$. Because there are three state variables, the state-transition diagram is a $3 \mathrm{D}$ matrix. We display each state $(n, t, b)$ and its neighbor states with the same value for $t$, using solid arrows in between; the only neighbors with a different $t$ value that can be reached directly from state $(n, t, b)$ are the states $(n, t-1, b)$ and $(n, t+1, b)$ 
(dashed arrows in Figure 2). This system can be modeled as a QBD where $n$ is the level and $(t, b)$ is the phase. Theorem 4 in Ramaswami and Lucantoni (1985) can be used to compute the waiting time distribution in the external queue from the steady state distribution of the QBD. A potential downside of this approach is that terms appearing in the infinite sum that expresses the waiting time distribution may decrease slowly when the uniformization parameter $\theta$ is large.

\subsection{Markov-modulated fluid queue}

A MMFQ is a two-dimensional Markov process $\left\{(L(\tau), \Phi(\tau)): \tau \in \mathbb{R}^{+}\right\}$, where $L(\tau)$ is the level at time $\tau$, and $\Phi(\tau)$ is the phase. The phase $\Phi(\tau)$ is an irreducible Markov process on a finite state space $\mathcal{U}$ that controls the rate at which the level varies. In general, the level varies linearly at rate $r_{\Phi(\tau)} \in \mathbb{R}$. If $L(\tau)=0$ and the rate is negative, the level remains at (i.e., it does not drop below) zero. For a detailed description of the MMFQ and its solution procedures, we refer readers to da Silva Soares (2005); Ramaswami (1999).

We model our system as a MMFQ $\left\{(X(\tau), \varphi(\tau)): \tau \in \mathbb{R}^{+}\right\}$where $X(\tau)$ is the waiting time of the first-in-line (FIL) patient in the external queue and phase $\varphi(\tau)=(n, t, b)$. The system will have different state space and transition rates at the boundary level $X(\tau)=0$.

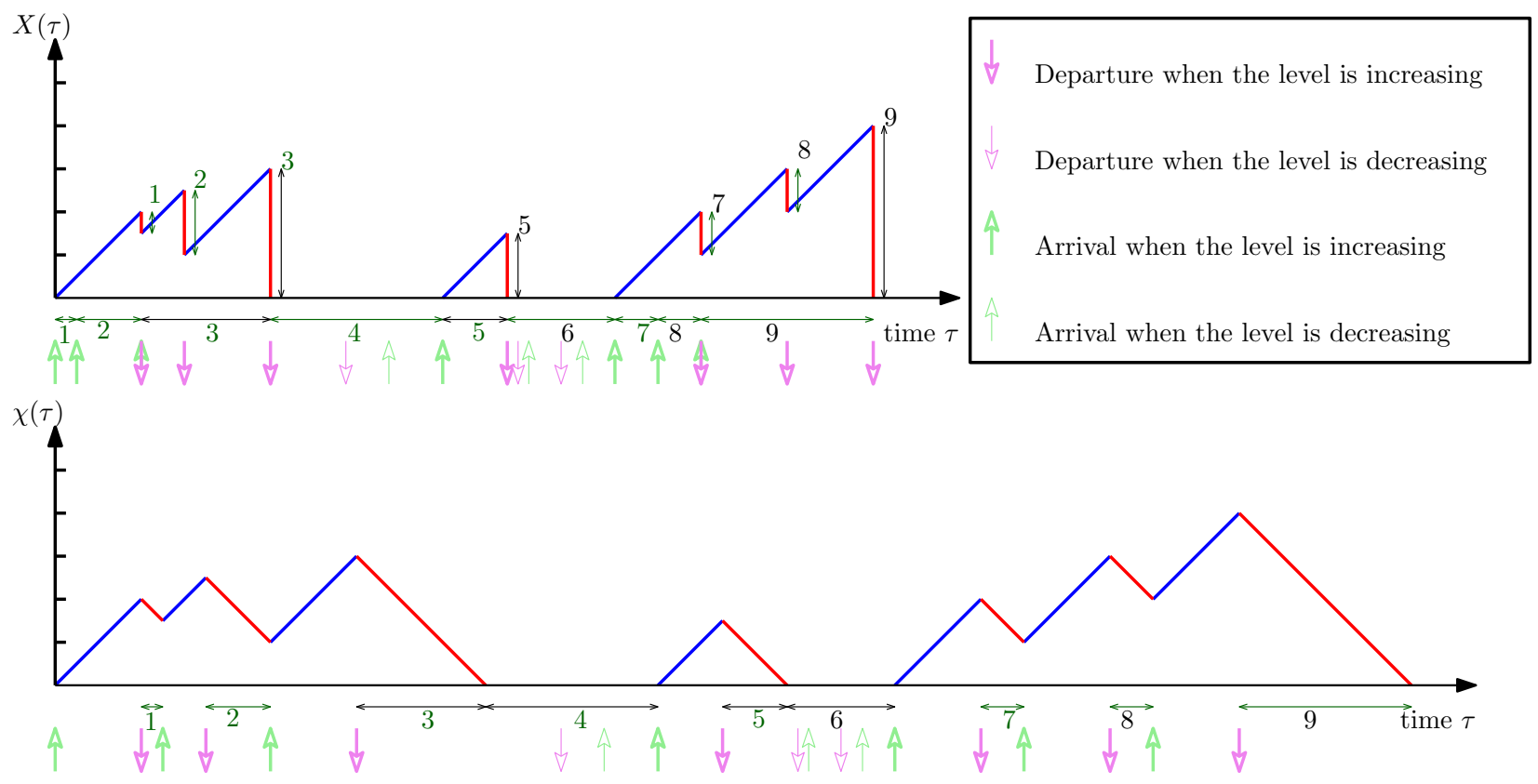

Figure 3: Evolution of the FIL patient's waiting time in the external queue (top) and the level of the associated fluid queue (bottom)

The top panel in Figure 3 shows a sample path of the FIL patient's waiting time in the external queue, as a function of time $\tau$. At $\tau=0$, all beds are occupied, and a new patient (patient 1 ) arrives in the external queue. His time in system increases steadily at rate 1 until a bed becomes available. At that time, patient 2 becomes the FIL patient. The level $X(\tau)$ thus decreases by the interarrival time between patients (indicated by the interval 1 in Figure 3). Meanwhile, the MMFQ phase is undergoing transitions: A patient is waiting in the external queue, so all $N$ beds are occupied by 
patients who may go from treatment to test or boarding while $X(\tau)$ changes. In the sample path of the bottom panel, all downward jumps of the top panel get replaced by time intervals in which the level decreases at a rate -1 ; the length of each interval is equal to the size of the corresponding downward jump. We denote this related fluid queue $\left\{(\chi(\tau), \phi(\tau)): \tau \in \mathbb{R}^{+}\right\}$. Because it only has rates $r_{\phi(\tau)}$ equal to -1 or +1 , it has elegant steady-state density expressions, which only need a minor adjustment to obtain the steady-state densities of the original jump process (da Silva Soares, 2005; da Silva Soares and Latouche, 2009, Dzial et al., 2005).

\subsubsection{Infinitesimal generator of $\phi(\tau)$.}

The state space $\mathcal{S}$ of $\phi(\tau)$ can be divided into two subsets because $\mathcal{S}=\mathcal{S}_{+} \cup \mathcal{S}_{-}$. The level's rate of change, $r_{\phi(\tau)}$, is +1 for $\phi(\tau)$ in $\mathcal{S}_{+}$, and -1 for $\phi(\tau)$ in $\mathcal{S}_{-}$. For $\chi(\tau)>0$, all $\mathrm{N}$ beds in the ED are occupied, and the infinitesimal generator of $\phi(\tau)$ is denoted by $\boldsymbol{T}$,

$$
\boldsymbol{T}=\left[\begin{array}{ll}
\boldsymbol{T}_{++} & \boldsymbol{T}_{+-} \\
\boldsymbol{T}_{-+} & \boldsymbol{T}_{--}
\end{array}\right]
$$

Both $\mathcal{S}_{+}$and $\mathcal{S}_{-}$hold states $(n, t, b)$ where $n \geq N$. Because the number of patients in the external queue is irrelevant for the evolution of the MMFQ, the phase of the system is completely defined by $(t, b)$; the size of state spaces $\left|\mathcal{S}_{+}\right|$and $\left|\mathcal{S}_{-}\right|$equals $\bar{m}=\frac{(N+1)(N+2)}{2}$. We explain the transition rates in each of the submatrices of $\boldsymbol{T}$ subsequently, note that $a=((N-t-b) \wedge s)$, as in Figure 2, $\quad \boldsymbol{T}_{++}$reflects the transition rates at which the phase changes while the FIL patient's waiting time increases. The phase moves within the state space $\mathcal{S}_{+}$and the level increases as long as there is a patient in the external queue and no bed becomes available. Therefore, $\boldsymbol{T}_{++}$is a square $(\bar{m} \times \bar{m})$ matrix with the following non-zero transition rates:

- Boarding after physician treatment at rate $\mu p_{b}(1-p) a:(t, b) \rightarrow(t, b+1)$.

- Tests after physician treatment at rate $\mu p a:(t, b) \rightarrow(t+1, b)$.

- Leaving tests at rate $t \delta:(t, b) \rightarrow(t-1, b)$.

- The diagonal contains negative rates such that the row sums of $\left[\begin{array}{lll}\boldsymbol{T}_{++} & \boldsymbol{T}_{+-}\end{array}\right]$are zero.

$\boldsymbol{T}_{--}$pertains to the rates at which the phase changes when the level decreases. The phase moves within the state space $\mathcal{S}_{-}$and, because of Poisson arrivals, $\boldsymbol{T}_{--}=-\lambda \boldsymbol{I}_{\overline{\boldsymbol{m}}}$.

$\boldsymbol{T}_{+-}$involves the rates at which the phase changes when a bed becomes available and the FIL patient enters this bed. The level changes from increasing to decreasing, and the phase moves from $\mathcal{S}_{+}$to $\mathcal{S}_{-}$. Because patients can leave the ED after treatment or after boarding, transition rates are $\mu(1-p)\left(1-p_{b}\right) a$ on the diagonal and $b \beta$ for transitions from $(t, b)$ to $(t, b-1)$.

$\boldsymbol{T}_{-+}$refers to the rates at which the phase changes from $\mathcal{S}_{-}$to $\mathcal{S}_{+}$. Since we are dealing with stationary Poisson arrivals, $\boldsymbol{T}_{-+}=\lambda \boldsymbol{I}_{\overline{\boldsymbol{m}}}$. 
For $\chi(\tau)=0$, departures and arrivals may occur while the level remains at zero (see, for example, periods 4 and 6 in Figure 3). For $\chi(\tau)=0$, the infinitesimal generator of $\phi(\tau)$ is denoted by $\boldsymbol{T}^{(0)}$;

$$
T^{(0)}=\left[\begin{array}{ll}
T_{-+}^{(0)} & T_{--}^{(0)}
\end{array}\right]
$$

where $\boldsymbol{T}_{--}^{(\mathbf{0})}$ pertains to the rates at which the phase changes as long as the external queue stays empty:

$$
\boldsymbol{T}_{--}^{(0)}=\left[\begin{array}{cc}
\boldsymbol{B}_{\mathbf{0}} & \boldsymbol{C}_{\mathbf{0}} \\
\boldsymbol{A}_{\mathbf{0}} & \boldsymbol{T}_{++}^{-\lambda \boldsymbol{I}_{\bar{m}}}
\end{array}\right] \text {. The phase changes within the state space } \mathcal{S}_{-}^{(0)}, \text { which is of size }
$$
$\bar{m}+\underline{m}$, with $\underline{m}=\sum_{i=0}^{N-1} \frac{(i+1)(i+2)}{2}$. Then, $\boldsymbol{T}_{--}^{(\mathbf{0})}$ pertains to the following components:

- $\boldsymbol{B}_{\mathbf{0}}$ is a square $(\underline{m} \times \underline{m})$ matrix that spans all states $(n, t, b)_{\{n<N, t+b \leq n\}}$ in lexicographic order. The matrix has the following non-zero transition rates:

- Arrivals at rate $\lambda:(n, t, b) \rightarrow(n+1, t, b)$.

- Departures after physician treatment at rate $\mu(1-p)\left(1-p_{b}\right) a:(n, t, b) \rightarrow(n-1, t, b)$.

- Boarding after physician treatment at rate $\mu p_{b}(1-p) a:(n, t, b) \rightarrow(n, t, b+1)$.

- Tests after physician treatment at rate $\mu p a:(n, t, b) \rightarrow(n, t+1, b)$.

- Departure after boarding at rate $b \beta:(n, t, b) \rightarrow(n-1, t, b-1)$.

- Leaving tests at rate $t \delta:(n, t, b) \rightarrow(n, t-1, b)$.

- The diagonal contains negative rates such that the row sums of $\left[\boldsymbol{B}_{\mathbf{0}}, \boldsymbol{C}_{\mathbf{0}}\right]$ are zero.

- $\boldsymbol{A}_{\mathbf{0}}$ is a matrix of size $(\bar{m} \times \underline{m})$. If there is at least one patient receiving physician treatment, transitions from state $(N, t, b)$ to $(N-1, t, b)$ occur at rate $\mu(1-p)\left(1-p_{b}\right) a$. If there is at least one boarding patient, transitions from state $(N, t, b)$ to $(N-1, t, b-1)$ occur at rate $b \beta$.

- $\boldsymbol{C}_{\mathbf{0}}$ is a matrix of size $(\underline{m} \times \bar{m})$. The system state changes from $(N-1, t, b)$ to $(N, t, b)$ at rate $\lambda$.

$\boldsymbol{T}_{-+}^{(\mathbf{0})}$ pertains to the rates at which the phase changes from state space $\mathcal{S}_{-}^{(0)}$ to $\mathcal{S}_{+}$. When there are exactly $\mathrm{N}$ patients in the system and a new patient arrives, the level becomes positive again (i.e., the last patient joins the external queue). Finally, $\boldsymbol{T}_{-+}^{(0)}=\left[\begin{array}{c}\mathbf{0}_{(\underline{m} \times \bar{m})} \\ \boldsymbol{T}_{-+}\end{array}\right]$.

\subsubsection{Stationary density vector of $\{(\chi(\tau), \phi(\tau))\}$ and $\{(X(\tau), \varphi(\tau))\}$.}

For any given level $x(x>0)$, the stationary density vector $\boldsymbol{\pi}(x)$ of $\{(\chi(\tau), \phi(\tau))\}$ has a matrix exponential form (Equation 3; see Dzial et al., 2005; Sengupta, 1989, 1990). Distinguishing between phases that feature increasing $\left(\boldsymbol{\pi}_{+}(x)\right)$ or decreasing $\left(\boldsymbol{\pi}_{-}(x)\right)$ levels, we obtain:

$$
\boldsymbol{\pi}(x)=\left[\boldsymbol{\pi}_{+}(x), \boldsymbol{\pi}_{-}(x)\right]=\left[\boldsymbol{p}_{-} \boldsymbol{T}_{-+}^{(\mathbf{0})} \exp (\boldsymbol{K} x), \boldsymbol{p}_{-} \boldsymbol{T}_{-+}^{(\mathbf{0})} \exp (\boldsymbol{K} x) \boldsymbol{\psi}\right]
$$


The matrix $\boldsymbol{K}$ can be computed using the iterative algorithm proposed in Sengupta (1989), which converges only linearly. Instead, we exploit the fact that downward jumps (Figure 3) have an exponential size (due to the Poisson arrivals); we construct a MMFQ, compute its steady state and censor out the periods where the fluid decreases to obtain the steady state of the original jump process. $\boldsymbol{K}=\boldsymbol{T}_{++}+\boldsymbol{\psi} \boldsymbol{T}_{-+}$where $\boldsymbol{\psi}$ is the solution of the algebraic Riccati equation in Equation 4 .

$$
\boldsymbol{T}_{+-}+\boldsymbol{T}_{++} \boldsymbol{\psi}+\boldsymbol{\psi} \boldsymbol{T}_{--}+\boldsymbol{\psi} \boldsymbol{T}_{-+} \psi=\mathbf{0}_{\overline{\mathbf{m}} \times \bar{m}}
$$

Efficient algorithms with quadratic convergence, such as the structure-preserving doubling algorithm (SDA, Guo et al., 2008) and the alternating directional doubling algorithm (Wang et al., 2012) are used to calculate $\boldsymbol{\psi}$ and $\boldsymbol{K}$. These algorithms significantly reduce computation times compared to the approach of Sengupta (1989), especially when the load is high. $\boldsymbol{p}_{-}$in Equation 3 is the stationary probability vector of level zero. It is the unique solution to $\boldsymbol{p}_{-}\left(\boldsymbol{T}_{--}^{(\mathbf{0})}+\boldsymbol{T}_{-+}^{(\mathbf{0})} \boldsymbol{\psi}^{(\mathbf{0})}\right)=\mathbf{0}_{(\mathbf{1} \times \overline{\boldsymbol{m}}+\underline{\boldsymbol{m}})}$, where $\boldsymbol{\psi}^{(\mathbf{0})}=\left[\begin{array}{ll}\mathbf{0}_{(\overline{\boldsymbol{m}} \times \underline{\boldsymbol{m}})} \quad \boldsymbol{\psi}\end{array}\right]$ Dzial et al. 2005$)$.

To obtain the stationary density of the original process $\{(X(\tau), \varphi(\tau))\}$, we censor $\{(\chi(\tau), \phi(\tau))\}$ by disregarding the phases with decreasing level (Dzial et al., 2005) and obtain:

$$
\begin{gathered}
\boldsymbol{\zeta}(x)=\alpha \boldsymbol{\pi}_{+}(x), \text { and } \\
\boldsymbol{\omega}=\alpha \boldsymbol{p}_{-} .
\end{gathered}
$$

The stationary density vector $\boldsymbol{\zeta}(x)_{\{x>0\}}$ is a row vector of length $\bar{m}$; each element $\boldsymbol{\zeta}_{t b}(x)$ denotes the joint probability that the FIL patient in the external queue has a waiting time of $x$, while the number of patients in the test (boarding) phase is $t(b)$. The row vector $\boldsymbol{\omega}$ is the stationary probability mass vector at level zero: It has length $\bar{m}+\underline{m}$, and each element $\omega_{n t b}$ denotes the probability that there are $n_{\{n \leq N\}}$ patients in the ED, of which $t$ are undergoing tests and $b$ are boarding (with $t+b \leq n$ ). The factor $\alpha$ can be obtained through normalization: $\boldsymbol{\omega} \mathbf{1}_{\overline{\mathbf{m}}+\underline{\boldsymbol{m}}}+\int_{0}^{\infty} \boldsymbol{\zeta}(x) \mathbf{1}_{\overline{\boldsymbol{m}}} d x=1$.

\subsubsection{Stability conditions.}

The MMFQ has a unique stationary distribution only if the network is stable. The stationary probability row vector $\boldsymbol{\xi}$ of rate matrix $\boldsymbol{T}$ is the unique solution of the system:

$$
\left\{\begin{array}{l}
\xi T=0_{2 \bar{m}} \\
\xi 1_{2 \bar{m}}=1
\end{array}\right.
$$

Distinguishing between phases with increasing and decreasing rates, we can write $\boldsymbol{\xi}=\left(\boldsymbol{\xi}_{+}, \boldsymbol{\xi}_{-}\right)$. The MMFQ is stable if the mean stationary drift of the fluid queue is negative: $\boldsymbol{\xi}_{+} \mathbf{1}_{\overline{\boldsymbol{m}}}-\boldsymbol{\xi}_{-} \mathbf{1}_{\overline{\boldsymbol{m}}}<0$. If the mean stationary drift is zero or positive, the fluid queue is null recurrent or transient, respectively (da Silva Soares, 2005, Ramaswami, 1999). Alternatively, stability is ensured if the arrival rate $\lambda$ is strictly smaller than the maximum throughput of the ED, as we detail in Section 3.2 .3 . 


\subsection{Performance Measures}

\subsubsection{External queue.}

Because the Poisson Arrivals See Time Averages (PASTA) property applies to the external queue, the delay probability in this queue is equal to the steady state probability of having $N$ or more patients in the system. If $\mathbf{1}^{\prime}$ is a column vector in which the first $\underline{m}$ elements are 1 and the last $\bar{m}$ elements are 0 , then

$$
\operatorname{Pr}_{e, q}(\text { delay })=1-\operatorname{Pr}\left(W_{e, q}=0\right)=1-\boldsymbol{\omega} \mathbf{1}^{\prime}
$$

The steady state density of the waiting time in the external queue, for $\tau>0$, can be expressed as follows:

$$
f_{W_{e, q}}(\tau)=\frac{\boldsymbol{\zeta}(\tau) \boldsymbol{\eta}}{\int_{0}^{\infty} \boldsymbol{\zeta}(y) \boldsymbol{\eta} d y}=\frac{\boldsymbol{p}_{-} \boldsymbol{T}_{-+}^{(\mathbf{0})} \exp (\boldsymbol{K} \tau) \boldsymbol{\eta}}{\boldsymbol{p}_{-} \boldsymbol{T}_{-+}^{(\mathbf{0})}(-\boldsymbol{K})^{-1} \boldsymbol{\eta}}
$$

where $\boldsymbol{\eta}$ is a length $\bar{m}$ column vector containing the departure rates of patients from the ED for each of the states in $\mathcal{S}_{+}$. Consequently, $\boldsymbol{\eta}$ equals the row sums of $\boldsymbol{T}_{+-}$.

The average waiting time $E\left[W_{e, q}\right]$ and the expected number of patients waiting for a bed $E\left[L_{e, q}\right]$ can be obtained as follows:

$$
\begin{aligned}
E\left[W_{e, q}\right] & =\operatorname{Pr}_{e, q}(\text { delay }) \int_{0}^{\infty} y \frac{\boldsymbol{\zeta}(y) \boldsymbol{\eta}}{\int_{0}^{\infty} \boldsymbol{\zeta}(x) \boldsymbol{\eta} d x} d y=\operatorname{Pr}_{e, q}(\text { delay }) \frac{\boldsymbol{p}_{-} \boldsymbol{T}_{-+}^{(\mathbf{0})} \boldsymbol{K}^{-2} \boldsymbol{\eta}}{\boldsymbol{p}_{-} \boldsymbol{T}_{-+}^{(\mathbf{0})}(-\boldsymbol{K})^{-1} \boldsymbol{\eta}} \\
E\left[L_{e, q}\right] & =\lambda E\left[W_{e, q}\right] .
\end{aligned}
$$

The service level in the external queue also has a matrix exponential form:

$$
\begin{aligned}
\operatorname{Pr}\left(W_{e, q} \leq \tau\right) & =\operatorname{Pr}\left(W_{e, q}=0\right)+\operatorname{Pr}_{e, q}(\text { delay }) \frac{\int_{0}^{\tau} \boldsymbol{\zeta}(y) \boldsymbol{\eta} d y}{\int_{0}^{\infty} \boldsymbol{\zeta}(y) \boldsymbol{\eta} d y} \\
& =\boldsymbol{\omega} \mathbf{1}^{\prime}+\left(1-\boldsymbol{\omega} \mathbf{1}^{\prime}\right) \frac{\boldsymbol{p}_{-} \boldsymbol{T}_{-+}^{(\mathbf{0})}(-\boldsymbol{K})^{-1} \exp (\boldsymbol{K} \tau) \boldsymbol{\eta}-\boldsymbol{p}_{-} \boldsymbol{T}_{-+}^{(\mathbf{0})}(-\boldsymbol{K})^{-1} \boldsymbol{\eta}}{\boldsymbol{p}_{-} \boldsymbol{T}_{-+}^{(\mathbf{0})}(-\boldsymbol{K})^{-1} \boldsymbol{\eta}}
\end{aligned}
$$

The probability of having $i$ patients in the external queue is (see Appendix A):

$$
\operatorname{Pr}\left(L_{e, q}=i\right)=\alpha \boldsymbol{p}_{-} \boldsymbol{T}_{-+}^{(\mathbf{0})} \lambda^{i-1}\left(\lambda \boldsymbol{I}_{\overline{\boldsymbol{m}}}-\boldsymbol{K}\right)^{-i} \mathbf{1}_{\overline{\boldsymbol{m}}}
$$

\subsubsection{Physician queue and physician utilization.}

Each patient has probability $p$ to undergo a test after each treatment, so the number of tests per patient is negative binomially distributed $N B(1, p)$ with mean $\frac{p}{1-p}$. The average number of physician treatments per patient equals $\frac{1}{1-p}$.

By Little's law (Little and Graves, 2008), the expected number of patients in treatment $E\left[L_{\text {Treatment }}\right]$ 
and the utilization of the physicians $\rho_{\text {phys }}$ are

$$
\begin{aligned}
E\left[L_{\text {Treatment }}\right] & =\frac{\lambda}{\mu(1-p)}, \text { and } \\
\rho_{\text {phys }} & =\frac{\lambda}{s \mu(1-p)} .
\end{aligned}
$$

We can denote $\varrho=\int_{0}^{\infty} \boldsymbol{\zeta}(x) d x=\alpha \boldsymbol{p}_{-} \boldsymbol{T}_{-+}^{(\mathbf{0})}(-\boldsymbol{K})^{-1}$, a row vector of length $\bar{m}$ in which $\varrho_{t b}$ is the joint probability that there is at least one patient in the external queue and there are $t$ test and $b$ boarding patients. The expected number of patients $E\left[L_{p h y s, q}\right]$ and average waiting time $E\left[W_{p h y s, q}\right]$ in the physician queue can be calculated as follows:

$$
\begin{aligned}
E\left[L_{\text {phys }, q}\right] & =\sum_{j=0}^{N} \sum_{k=0}^{N-j}((N-j-k-s) \vee 0) \varrho_{j k}+\sum_{i=0}^{N} \sum_{j=0}^{i} \sum_{k=0}^{i-j}((i-j-k-s) \vee 0) \omega_{i j k} \\
E\left[W_{\text {phys }, q}\right] & =\frac{(1-p) E\left[L_{\text {phys }, q}\right]}{\lambda}
\end{aligned}
$$

Because of the limited number of beds, the physician queue will not have a stationary Poisson arrival process, and PASTA does not apply. The probability $q_{j}$ that an arrival to the physician queue finds $j$ patients waiting for or with a physician is given by (e.g., Gross and Harris, 1985):

$$
\begin{aligned}
& q_{j(j \in\{0, \ldots, N-1\})}=c \cdot\left[\sum_{b=0}^{N-j} \varrho_{N-j-b, b}((N-j-b) \delta+b \beta)\right. \\
& +\sum_{b=0}^{N-j-1} \varrho_{N-j-b-1, b}\left((s \wedge j+1) \mu(1-p)\left(1-p_{b}\right)\right) \\
& \left.+\sum_{b=0}^{n-j} \sum_{n=j}^{N} \omega_{n, n-j-b, b}((n-j-b) \delta+[n<N] \lambda)\right]
\end{aligned}
$$

where $c$ is a normalization constant that ensures that $\sum_{j} q_{j}=1$, and the notation $[A]$ on the last line denotes the Iverson bracket, yielding 1 if $\mathrm{A}$ is true and 0 if $\mathrm{A}$ is false.

The waiting time in the physician queue, given that $j_{\{j \geq s\}}$ patients are waiting for or with the physician, has an Erlang distribution with $j-s+1$ stages and rate $s \mu$ (Kleinrock, 1975). Therefore, the service level in the physician queue can be calculated as:

$$
\begin{aligned}
\operatorname{Pr}\left(W_{\text {phys }, q} \leq \tau\right) & =\operatorname{Pr}\left(W_{\text {phys }, q}=0\right)+\sum_{j=s}^{N-1} q_{j} \cdot \int_{0}^{\tau} \frac{s \mu(s \mu x)^{j-s}}{(j-s) !} \exp (-s \mu x) d x \\
& =1-\sum_{j=s}^{N-1} q_{j} \cdot \sum_{z=0}^{j-s} \frac{(s \mu \tau)^{z}}{z !} \exp (-s \mu \tau) .
\end{aligned}
$$




\subsubsection{LOS, patient census and maximum throughput.}

The total expected length of stay (excluding boarding, Equation 17) includes the average waiting times, the average total treatment time, and the average total test time. Alternatively, it can be expressed as the difference between the total average time in the system and the average boarding time. That is,

$$
\begin{aligned}
E[L O S] & =E\left[W_{e, q}\right]+\frac{E\left[W_{p h y s, q}\right]}{(1-p)}+\frac{1}{\mu(1-p)}+\frac{p}{\delta(1-p)} \\
& =\frac{E[L]}{\lambda}-\frac{p_{b}}{\beta} .
\end{aligned}
$$

The expected total patient census can be calculated as

$$
E[L]=E\left[L_{b e d}\right]+E\left[L_{e, q}\right]
$$

where $E\left[L_{b e d}\right]$ refers to the expected number of occupied beds:

$$
\begin{aligned}
E\left[L_{b e d}\right] & =N \varrho \mathbf{1}_{\overline{\mathbf{m}}}+\sum_{i=0}^{N} \sum_{j=0}^{i} \sum_{k=0}^{i-j} i \omega_{i j k}, \text { and } \\
\rho_{\text {bed }} & =\frac{E\left[L_{b e d}\right]}{N} .
\end{aligned}
$$

The expected number of boarding patients follows from $E\left[L_{\text {Boarding }}\right]=\frac{p_{b} \lambda}{\beta}$; the expected number of patients undergoing tests equals $E\left[L_{\text {Test }}\right]=\frac{p \lambda}{\delta(1-p)}$.

The maximum throughput ( $M a x T H$ ) of the ED equals the throughput of the equivalent closed system with $N$ patients (Figure 4 ). It is calculated as

$$
\operatorname{Max} T H=\sum_{p h=0}^{N-b} \sum_{b=0}^{N} \pi_{p h, b}\left((1-p)\left(1-p_{b}\right) \mu(s \wedge p h)+\beta b\right) .
$$

where $\pi_{p h, b}$ denotes the steady state probability of having $p h$ patients undergoing physician treatment and $b$ boarding patients. The network in Figure 4 is a product-form closed Jackson network with $\mathrm{N}$ patients, so these probabilities can be obtained easily (for an extensive review of solution procedures for closed networks, see Lagershausen, 2012). As mentioned in Section 3.1.3, the external queue is stable only if the arrival rate $\lambda$ is strictly smaller than Max TH.

\section{Computational Results}

In this section, we analyze the impact of the boarding parameters on ED congestion and ED capacity. We introduce the experimental settings in Section 4.1, then analyze the results in Section 4.2 , 


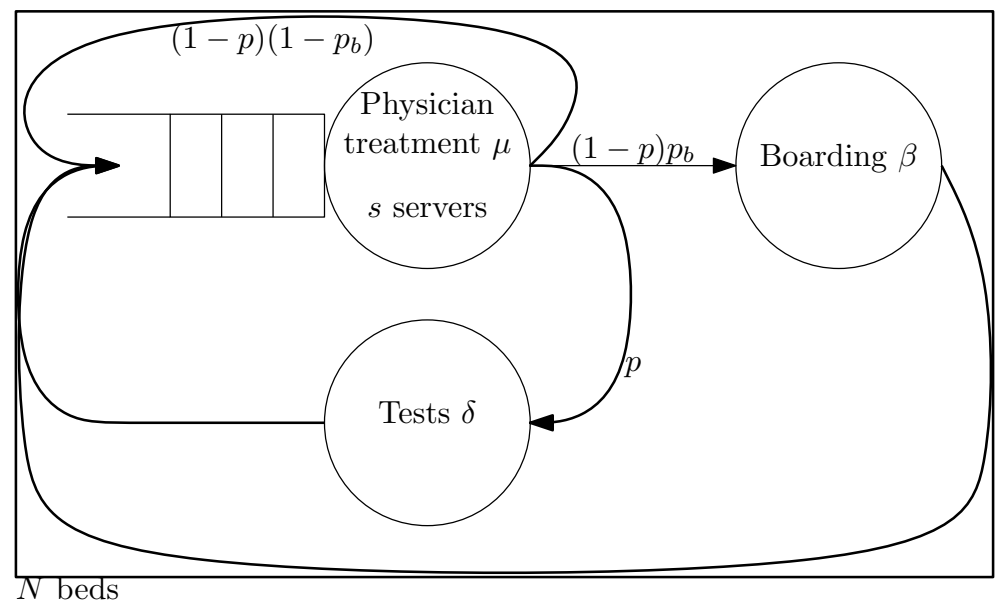

Figure 4: Corresponding closed model

\begin{tabular}{lcccccccccc}
\hline & $\mu$ & $\delta$ & $p$ & $p_{b}$ & $\beta$ & $\lambda$ & $s$ & $N$ & $\rho_{\text {phys }}$ & $\rho_{\text {bed }}$ \\
\hline HL & 12.37 & 1.310 & 0.7268 & $35 \%$ & $\frac{1}{3}$ & 5.4 & 2 & 25 & $79.89 \%$ & $81.35 \%$ \\
LL & 12.37 & 1.310 & 0.7268 & $35 \%$ & $\frac{1}{3}$ & 2.7 & 1 & 25 & $79.89 \%$ & $48.70 \%$ \\
\hline
\end{tabular}

Table 2: Inputs for the base case scenario (all rates are expressed in patients per hour)

\subsection{Experimental Setting}

As observed by Armony et al. (2015) and Wiler et al. (2013), the daily operation of an ED can be roughly split into a peak period and an off-peak period, during which steady-state-like behavior can be observed. We therefore conduct experiments under high-load (HL) and low-load (LL) conditions (see Table 2, all rates in this table are expressed in patients per hour). The number of beds remains constant over the day, so the external queue diminishes during the LL period, when the system will behave almost like an open queueing system. During the HL period, beds are a scarce resource, and the semi-open nature of the network affects performance. To calibrate the model based on historical data, it is recommended to distinguish and estimate separate arrival rates for at least two periods; one high load during the day and one low load during the night. Our inputs for the experiments are based on values reported in prior healthcare and operations management literature. The estimates for $\mu, \delta$, and $p$ come from Yom-Tov and Mandelbaum (2014), who obtained these from real data. Based on Armony et al. (2015), we set boarding probability $p_{b}$ equal to $35 \%$ and boarding rate $\beta$ to $\frac{1}{3}$ (consistent with what other researchers have observed; see Carmen et al. 2015; Lucas et al. 2009; Shi et al. 2015). In the HL case, the arrival rate $\lambda$ is chosen such that $\rho_{\text {phys }}$ is close to $80 \%$ (Equation 13, and the number of beds is chosen such that $\rho_{\text {bed }}$ is close to $80 \%$. In the LL case, the reduced $\lambda$ reflects the lower influx of patients, and the number of doctors decreases from 2 to 1 , such that doctor utilization stays close to $80 \%$. The number of beds remains the same though, so bed utilization in the LL case evidently decreases. To analyze the impact of the boarding parameters on ED congestion and ED capacity, we analyze the system for varying values of $\beta, p_{b}$, and $N$. 


\subsection{Results: Impact of boarding parameters on ED performance}

By occupying beds, boarding patients may delay the start of treatment for newly arriving patients, thereby influencing the external and internal queues.



(a) Scenario HL

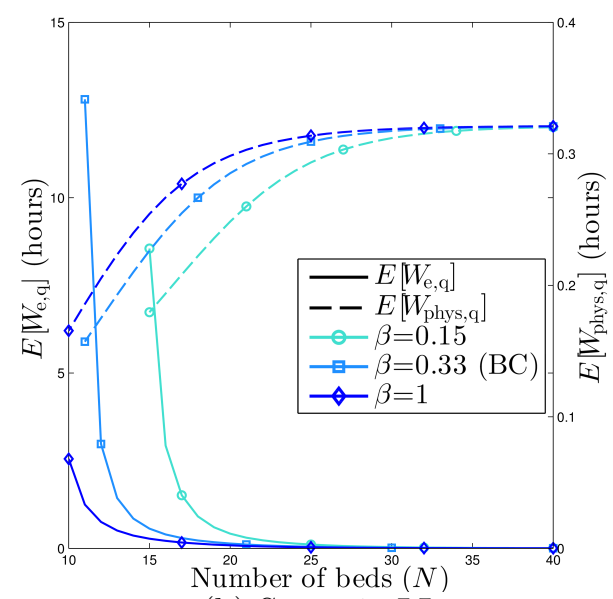

(b) Scenario LL

Figure 5: Impact of $\beta$ on $E\left[W_{e, q}\right]$ and $E\left[W_{p h y s, q}\right]$, for varying values of $N$

Figure 5 shows the impact of changes in $\beta$ on the expected waiting time in the external and physician queues $\left(E\left[W_{e, q}\right]\right.$, resp. $\left.E\left[W_{\text {phys,q }}\right]\right)$ for varying values of $N$. For example, $\beta$ could increase if the ED improved the pre- and post-allocation delays of admitted patients (Shi et al., 2015); alternatively, the hospital could influence $\beta$ by "pushing" patients out of the ED toward the IW when the boarding problem becomes severe (Greene, 2007) (we investigate the impact of such a policy in Section 5.1).

As is evident from Figure 5, lower $\beta$ values increase the external queue waiting time, for any given number of beds; this finding is intuitive as both the number of boarding patients and bed utilization increase. Consequently, lower $\beta$ values require more beds to keep the external queue stable. This effect is more pronounced in the HL case than in the LL case. Figure 5 also reveals that a decreased $\beta$ leads to a decrease in the physician queue waiting time. This outcome is not due to a decrease in physician utilization; as evident from expression 13 , physician utilization is independent of $\beta$. Yet as the number of boarding patients increases, fewer patients remain who can simultaneously call on physicians for service, so the waiting time in the physician queue reduces. Figure 5 reveals that the impact of $\beta$ on $E\left[W_{e, q}\right]$ and $E\left[W_{p h y s, q}\right]$ vanishes as the number of beds $N$ increases. This result again is intuitive: As the number of beds grows, boarding patients less frequently prevent needy patients from gaining access to beds, and the system increasingly acts as an open queueing network instead of a semi-open queueing network. As $E\left[W_{e, q}\right]$ decreases, $E\left[W_{p h y s, q}\right]$ goes up, because the larger number of beds is increasingly occupied by needy patients. Eventually, for sufficiently large $N$, the system effectively behaves as an open queueing network, and both queues are hardly influenced by the boarding effects. Note that $E\left[W_{\text {phys,q }}\right]$ stabilizes at a higher level in the LL case; only one physician is available, so the LL system cannot benefit from resource pooling effects.

Figure 6 shows the impact of a change in $\beta$ on the service level in the external queue: $\operatorname{Pr}\left(W_{e, q} \leq\right.$ $\tau)$, for $\tau=0$ or 30 minutes. As $\beta$ decreases, this service level diminishes. In the HL setting, this 


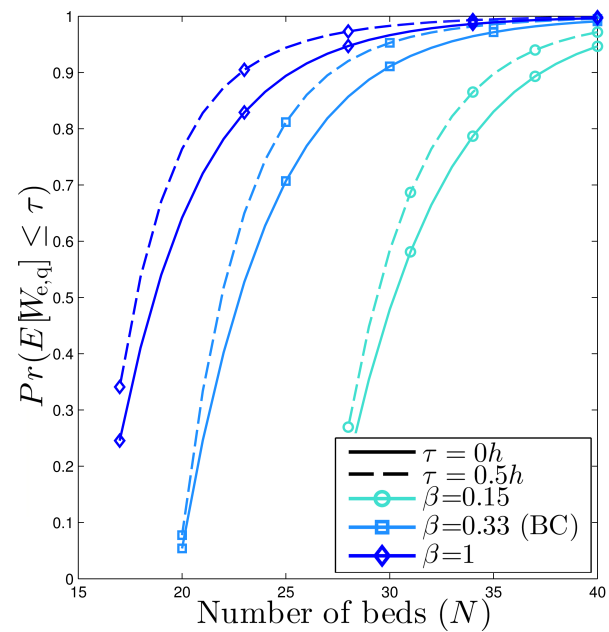

(a) Scenario HL

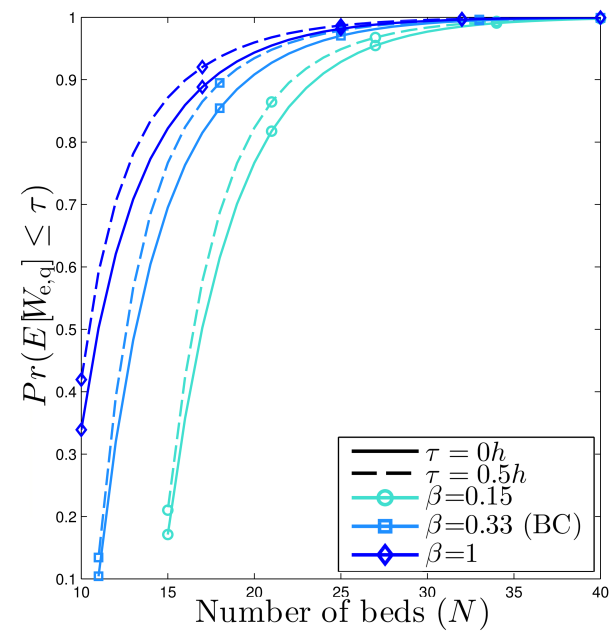

(b) Scenario LL

Figure 6: Effect of $\beta$ on the service level in the external queue, for varying values of $N$

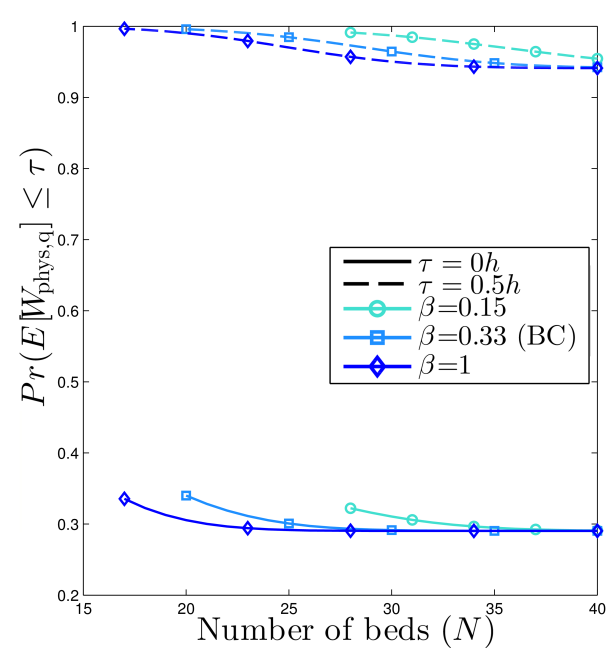

(a) Scenario HL

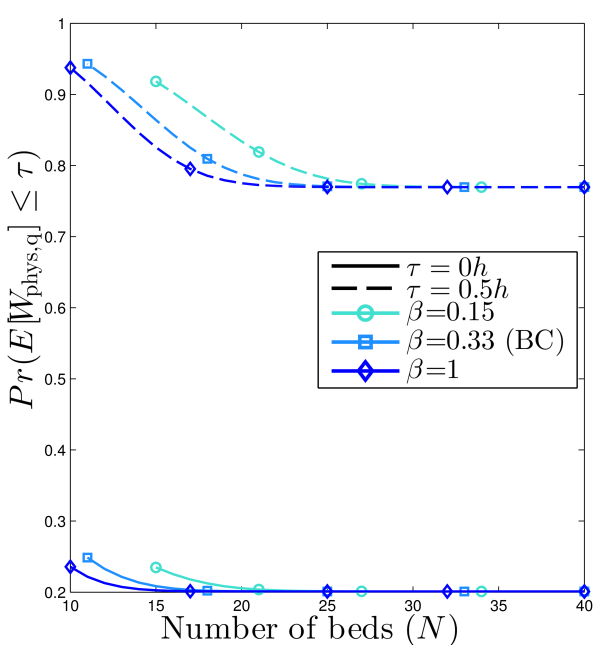

(b) Scenario LL

Figure 7: Effect of $\beta$ on the service level in the physician queue, for varying values of $N$

impact is much more severe than in the LL setting, reflecting that beds are a tighter bottleneck under a high load. Figure 7 shows the impact on the service level in the physician queue. The service levels are clearly lower in the LL setting, because there are fewer boarding patients occupying the available beds. For sufficiently large values of $N$, the service level is no longer influenced by the boarding rate. As noted previously, the system acts as an open system, and the service level in the physician queue reaches a minimum.

Surprisingly, two systems with identical $E\left[L_{\text {boarding }}\right]$ but different values for $p_{b}$ and $\beta$ might result in very different performance, especially when bed utilization is high. This is illustrated in Figures 8 to 10 : these curves represent the behavior of the base case scenario (Table 2 ) when we alter the level of $E\left[L_{\text {boarding }}\right]$. Along a given curve, the value of $E\left[L_{\text {boarding }}\right]$ is constant: on each curve, $\beta$ thus changes accordingly with $p_{b}, \beta=\frac{p_{b} \lambda}{E\left[L_{\text {boarding }}\right]}$. Note that the $\mathrm{y}$-axis on these curves represents the performance relative to the performance when $\beta=0.35$ (the base case) and there are $2,4,6$, or $8(3,9,11$, or 13$)$ boarding patients on average in the HL (LL) scenario. The dashed lines 
show performance when there are no boarding patients but $x$ beds are permanently unavailable. As Figure 8 demonstrates, congestion in the external queue increases as $E\left[L_{\text {boarding }}\right]$ increases, and the impact on congestion is worse when the boarding occurs infrequently (low $p_{b}$ values) and expected boarding times are long (low $\beta$ values). Indeed, the curves show an upswing as $p_{b}$ decreases. This upswing occurs as, under these conditions, the number of boarding patients will exhibit a higher variance; fewer patients have to board, but those who do, have to stay longer. As a consequence, the number of beds available for treatment will also vary significantly, and newly arriving patients are more likely to wait before obtaining a bed (as evident from Figure 9). The dashed lines show that $E\left[L_{e, q}\right]$ is significantly lower if a fixed number of beds are unavailable, instead of varying randomly due to boarding patients. Interestingly, these conditions also impact the physician queue, especially when bed utilization is high (HL case). As evident from Figure 10 , increasing $E\left[L_{\text {boarding }}\right]$ decreases

$E\left[L_{\text {phys,q }}\right]$, as observed previously; yet, congestion in the physician queue improves (slightly) as boarding becomes more irregular in nature (low $p_{b}$ values, going hand in hand with a decrease in $\beta$ ). Under these conditions, the physician stage will be starved more often: i.e., there is a higher probability that at least one physician is idle $(t<s)$ while patients wait in the external queue $(n>N)$. Evidently, this is negative for system performance, as it means that valuable physician time gets wasted due to bed shortage. For ease of reference, we include the curves for the starvation probability in Appendix B.

In summary, EDs that have (on average) the same number of boarding patients can have markedly different performance in both the external queue and the physician queue: those with low boarding rates suffer consistently worse behavior in the external queue, even when the boarding probability is low. High boarding probabilities are not as detrimental for the external queue, if they go together with high boarding rates. Note that high $\beta$ values are more likely to occur in hospitals with large IWs or flexible admission policies: e.g., if beds are unavailable in the primary ward, patients can be sent to secondary wards (referred to as department clustering Essen et al. 2014 or bed overflow Shi et al., 2013; Teow et al., 2011).

While the variability of the boarding process has a clear impact on the queue behavior in the ED, it has no impact on ED capacity $(\operatorname{Max} T H)$. As shown in Figure 11, Max TH only depends on $E\left[L_{\text {Boarding }}\right]$, regardless of the values of $p_{b}$ and $\beta$. As $E\left[L_{\text {Boarding }}\right]$ increases, Max $T H$ goes down; this trend is more evident in the HL case, because beds present a larger impediment to the physicians' treatment in that case.

\section{Reducing boarding in the ED: Reactive versus proactive interventions}

This section discusses two types of strategies to reduce boarding. In the first, reactive strategy (Section 5.1), the IW is notified whenever the number of boarding patients exceeds a threshold, so the signal urges the IW to increase $\beta$ for as long as overcrowding in the ED persists. The second, proactive strategy (Section 5.2) reserves some set number of beds (buffer beds) for patients who need to be admitted. Section 5.3 compares the impact of both strategies on ED performance. 




(a) Scenario HL

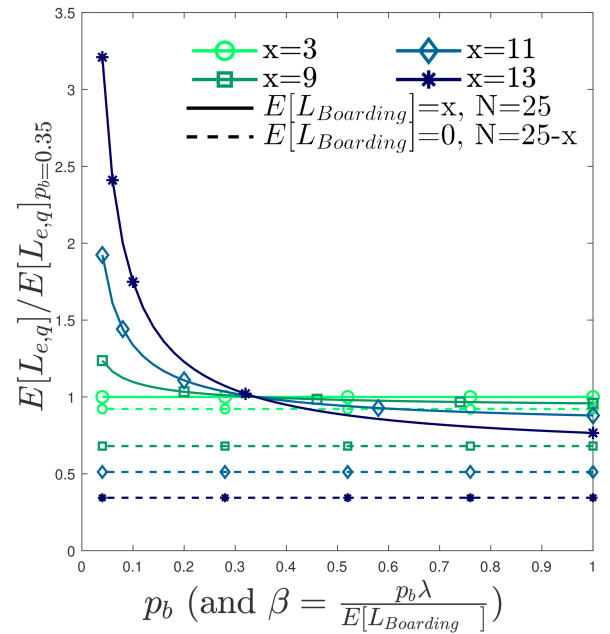

(b) Scenario LL

Figure 8: Effect of changes in $\beta$ and $p_{b}$ on $E\left[L_{e, q}\right]$

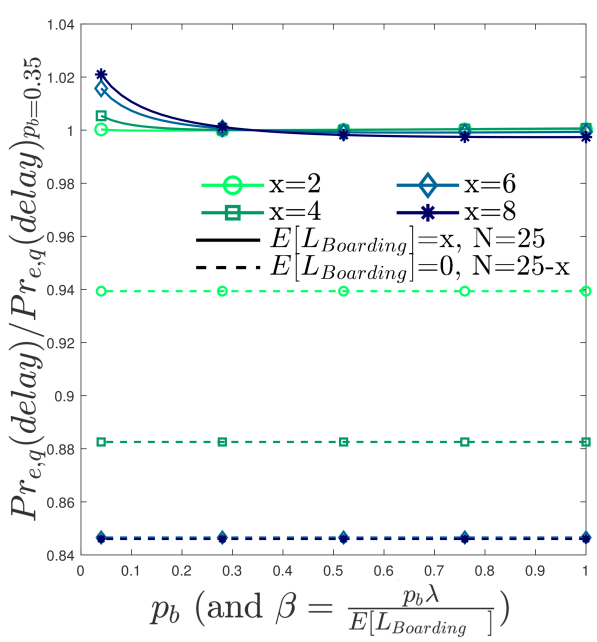

(a) Scenario HL

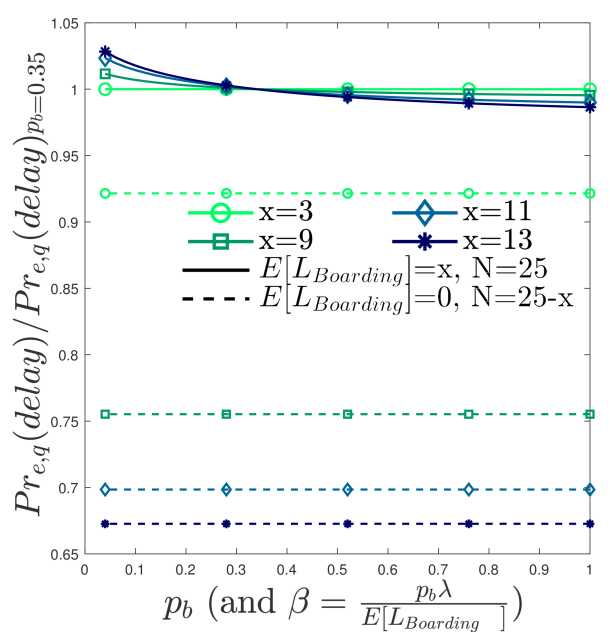

(b) Scenario LL

Figure 9: Effect of changes in $\beta$ and $p_{b}$ on $\operatorname{Pr}_{e, q}$ (delay) 




(a) Scenario HL

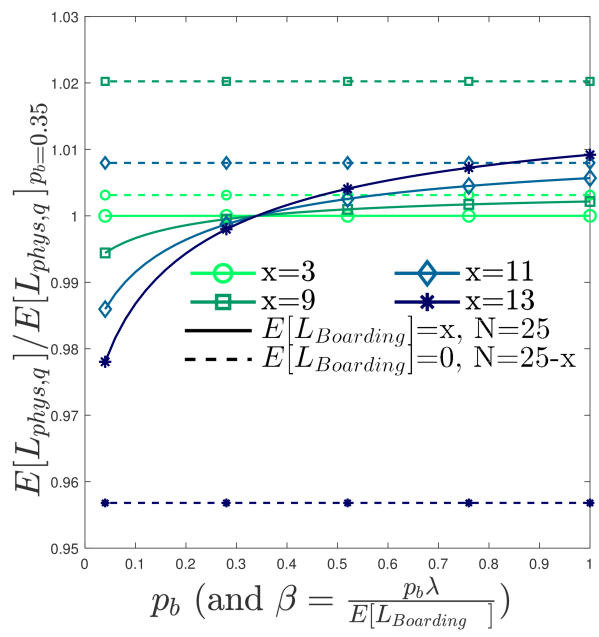

(b) Scenario LL

Figure 10: Effect of changes in $\beta$ and $p_{b}$ on $E\left[L_{P h y s, q}\right]$

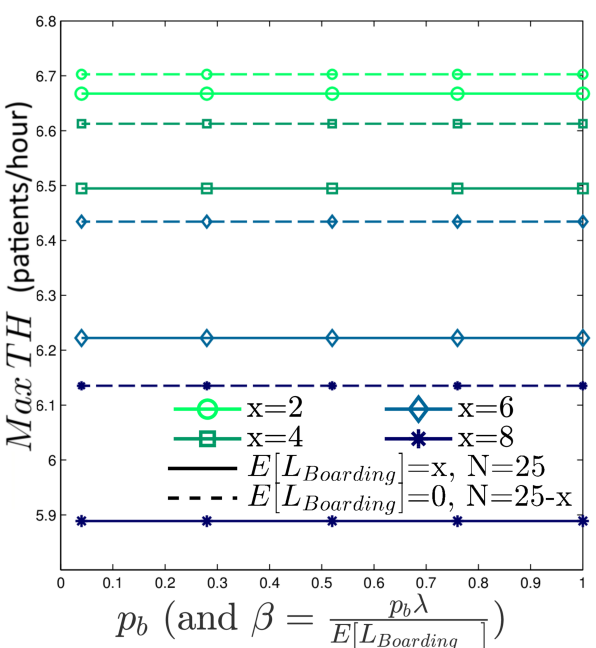

(a) Scenario HL



(b) Scenario LL

Figure 11: Effect on $\operatorname{Max} T H$ 


\subsection{Reactive policy: State-dependent boarding rate}

In practice, a reactive policy that urges the IW to take ED patients in faster whenever the number of boarding patients exceeds a threshold is often implemented by moving patients to inpatient hallways or discharging patients earlier (Chan et al., 2012; Greene, 2007; Kelen et al., 2006. Thompson et al., 2009; Viccellio et al., 2009). Villa-Roel et al. (2012) review the implementation of these kinds of full capacity protocols in practice. Methodologically, we implement the reactive policy by increasing the boarding rate $\beta$ by a speedup factor $\alpha>1$ when the number of boarding patients reaches a threshold $\theta$. The transition rate from state $(n, t, b)$ to $(n-1, t, b-1)$ thus equals $b \beta$ for $b<\theta$ and $b \alpha \beta$ for $b \geq \theta$. The size of the state space and the time to calculate the steady state probabilities are not affected.

\subsection{Proactive policy: Dedicated beds for boarding patients}

This strategy provides a limited number of beds buffer beds outside the ED for patients waiting to be admitted, such as in so-called acute medical admission units or holding areas (Kolb et al., 2008); see van der Linden et al. (2013) and Viccellio et al. (2013) for alternative implementation options. We assume that patients that need to be admitted are sent to one of these $B$ buffer beds; only when there are more than $B$ patients waiting for admission, patients still need to board in one of the $N$ ED beds.

The number of boarding patients now can exceed the number of ED beds, so the state space is equal to the set of triplets $\{(n, t, b) \mid n \in\{0,1, \ldots, \infty\}, t \in\{0,1, \ldots, N\}, b \in\{0,1, \ldots, N+B\}$, with $t+b \leq$ $n \wedge(N+b \wedge B)\}$, and where $n$ now represents the total number of patients in the ED and the buffer beds. The size of $\left|\mathcal{S}^{0}\right|,\left|\mathcal{S}_{-}\right|$, and $\left|\mathcal{S}_{+}\right|$thus increases slightly.

\subsection{Comparison of queue behavior and service levels}

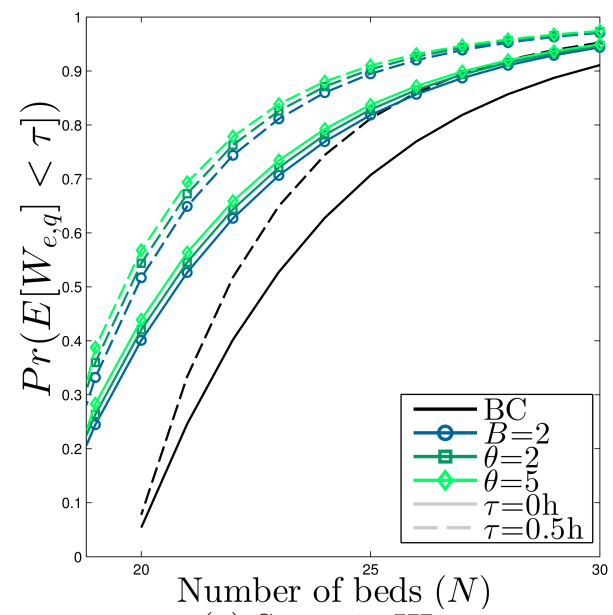

(a) Scenario HL



(b) Scenario LL

Figure 12: Effect of a reactive or proactive policy on the external queue service level for different values of $N$

Figure 12 shows the impact on the external queue service level when implementing a proactive policy with two buffer beds versus a reactive policy that is equivalent. We regard two policies as 


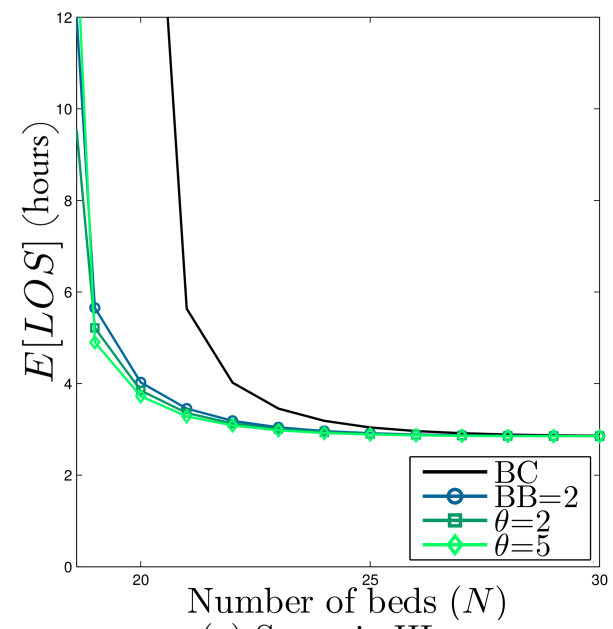

(a) Scenario HL

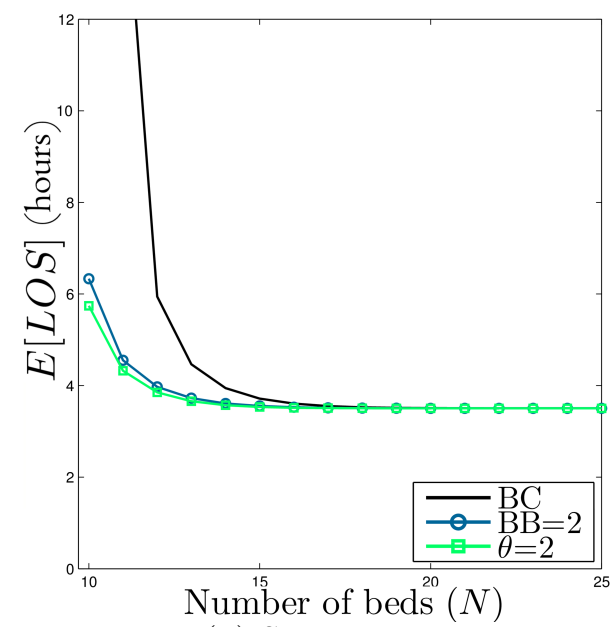

(b) Scenario LL

Figure 13: Effect of a reactive or proactive policy on $E[L O S]$ for different values of $N$

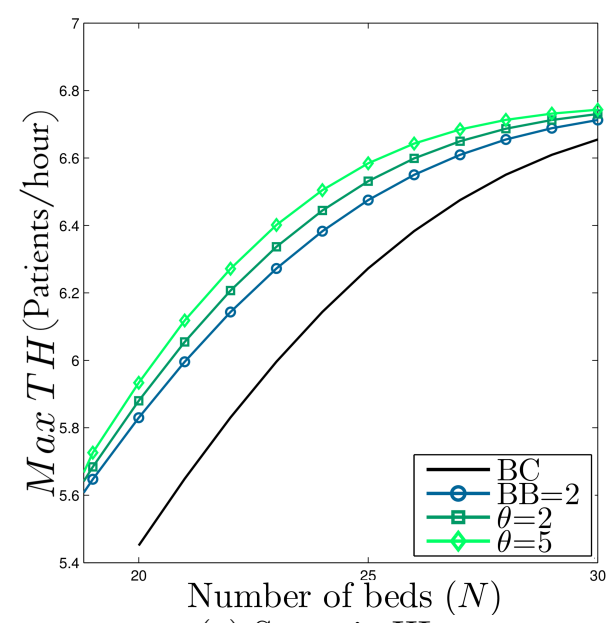

(a) Scenario HL

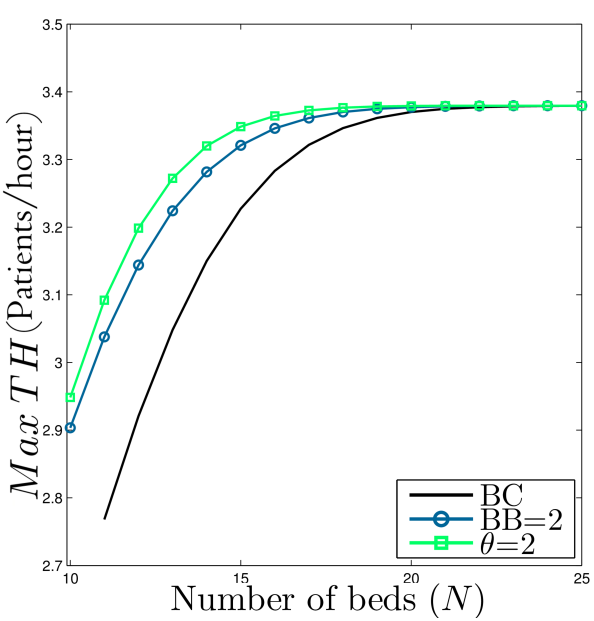

(b) Scenario LL

Figure 14: Effect of a reactive or proactive policy on $M a x T H$ for different values of $N$

equivalent if they result in the same expected number of boarding patients occupying ED beds. Note that, in the proactive policy, boarding patients may also reside in buffer beds, so they do not necessarily block any ED beds. To find the equivalent reactive policy for an arbitrary proactive policy, we adjust the speedup factor $\alpha$ for each $N$ and each $\theta$; as the speedup factor is limited in practice, we allow a maximum $\alpha$ value of 6 . For high $\theta$ or high $B$ values, the equivalent reactive policy thus may not exist. For ease of interpretation, this section only compares the results assuming a proactive policy with $B=2$ buffer beds; the insights remain valid also for other values of $B$.

It is clear from Figure 12 that the reactive policies lead to higher service levels in the external queue, compared to the proactive policy. This observation can be linked to the discussion in Section 4.2, in which we observed that, ceteris paribus, performance improves more when boarding is reduced by acting on $\beta$, rather than on $p_{b}$. Indeed, the reactive policies increase $\beta$ (when $\theta$ is exceeded), while the proactive policy implicitly reduces the probability that an ED bed is needed for a boarding patient $\left(p_{b}\right)$. The increase in $\beta$ in the reactive policies brings down the number of boarding patients fast (the higher the threshold, the greater the speedup), and the external queue 
will not have time to build up. Appendix C shows the density function for the number of ED beds occupied by boarding patients; clearly, the reactive policy not only reduces the expected number of such beds, but also limits the variance, when compared to the proactive policy. Though a decrease in boarding comes with a minor increase in congestion in the physician queue (as discussed in Section 4.2), the introduction of boarding reduction policies reduces the overall expected length of stay of patients in the ED $(E[L O S])$, as shown in Figure 13). The reactive policy also substantially increases the capacity of the ED, and outperforms the increase obtained with the proactive policy; see Figure 14. This indicates that the reactive policy will be able to cope with higher arrival rates.

In general, we can thus conclude that, from an operational perspective, reactive policies should be preferred over proactive policies; interestingly, they are also likely to score best from a cost perspective, as they do not explicitly require extra resources (extra beds, personnel, etc.).

\section{Conclusions}

This article uses a MMFQ approach to gain insights into the effect of patient boarding on ED performance. Our analysis is numerical and exact; it is also computationally faster than the QBD method for the performance measures considered, especially for calculating service levels.

Although boarding patients only occupy beds, our results show that their influence on ED congestion and maximum ED capacity may be substantial, especially when the utilization of ED beds is high. We also observe that patient boarding caused by low admission rates has a worse effect on the external queue than patient boarding caused by high boarding probabilities. Boarding reduction policies are influenced by this effect; a reactive policy that reduces the boarding time when the number of boarding patients exceeds a threshold outperforms a proactive policy in which some beds are reserved outside the ED for patients as they wait for admission.

There are some limitations to our study. We assume homogeneous patients, treated according to a first come, first served policy. In reality, patients are treated according to their urgency, and high-priority patients may preempt the service of a low-priority patient. Boarding thus mainly affects the delays of lower priority patients in practice, and they likely benefit most from boarding reduction policies. Also, we do not incorporate time-of-day dependence in boarding behavior (Shi) et al. 2015), and we assume that boarding patients only occupy ED beds and do not need ED staff. In practice, ED staff generally remain responsible for these patients as long as they reside in the ED (Armony et al., 2015). Finally, we do not include abandonments; abandonments from the external queue could be incorporated, though, by using a level-dependent MMFQ (for an example, see Horváth and Van Houdt, 2012).

\section{Acknowledgments}

This research was supported by the Research Foundation-Flanders (FWO) (grant $\mathrm{n}^{\circ}$ G.0768.15). 


\section{Bibliography}

Armony, M., Israelit, S., Mandelbaum, A., Marmor, Y. N., Tseytlin, Y., Yom-Tov, G. B., et al., 2015. On patient flow in hospitals: A data-based queueing-science perspective. Stochastic Systems 5 (1), 146-194.

Bernstein, S. L., Aronsky, D., Duseja, R., Epstein, S., Handel, D., Hwang, U., McCarthy, M., John McConnell, K., Pines, J. M., Rathlev, N., Schafermeyer, R., Zwemer, F., Schull, M., Asplin, B. R., Society for Academic Emergency Medicine, E. D. C. T. F., 2009. The effect of emergency department crowding on clinically oriented outcomes. Academic Emergency Medicine 16 (1), 1-10.

URL http://dx.doi .org/10.1111/j.1553-2712.2008.00295.x

Buitenhek, R., van Houtum, G.-J., Zijm, H., 2000. AMVA-based solution procedures for open queueing networks with population constraints. Annals of Operations Research 93 (1-4), 15-40.

URL http://dx.doi.org/10.1023/A\%3A1018967622069

Campello, F., Ingolfsson, A., Shumsky, R. A., 2016. Queueing models of case managers. Management Science Articles in Advance, 1-19.

URL http://dx.doi.org/10.1287/mnsc.2015.2368

Carmen, R., Defraeye, M., Van Nieuwenhuyse, I., 2015. A decision support system for capacity planning in emergency departments. International Journal of Simulation Modelling 14 (2), 299-312.

Chan, C. W., Farias, V. F., Bambos, N., Escobar, G. J., 2012. Optimizing intensive care unit discharge decisions with patient readmissions. Operations Research 60 (6), 1323-1341. URL http://pubsonline .informs .org/doi/abs/10.1287/opre.1120.1105

da Silva Soares, A., March 2005. Fluid queues: building upon the analogy with QBD processes. Ph.D. thesis, Université Libre de Bruxelles.

da Silva Soares, A., Latouche, G., 2009. Fluid queues with level dependent evolution. European Journal of Operational Research 196 (3), 1041-1048.

URL http://www. sciencedirect.com/science/article/pii/S0377221708004165

Dallery, Y., 1990. Approximate analysis of general open queuing networks with restricted capacity. Performance Evaluation 11 (3), 209-222.

URL http://www.sciencedirect.com/science/article/pii/0166531690900139

de Véricourt, F., Jennings, O. B., 2011. Nurse staffing in medical units: A queueing perspective. Operations Research 59 (6), 1320-1331.

URL http://dx.doi.org/10.1287/opre.1110.0968

Dhingra, V., Kumawat, G. L., Roy, D., de Koster, R., 2018. Solving semi-open queuing networks with timevarying arrivals: An application in container terminal landside operations. European Journal of Operational Research 267 (3), $855-876$.

URL http://www.sciencedirect.com/science/article/pii/S0377221717311396

Dzial, T., Breuer, L., da Silva Soares, A., Latouche, G., Remiche, M.-A., Oct. 2005. Fluid queues to solve jump processes. Performance Evaluation 62 (1-4), 132-146.

URL http://dx.doi.org/10.1016/j.peva.2005.07.013

Essen, J. T., Houdenhoven, M., Hurink, J. L., 2014. Clustering clinical departments for wards to achieve a prespecified blocking probability. OR Spectrum 37 (1), 243-271.

URL http://dx.doi.org/10.1007/s00291-014-0368-5

Green, L., 2006. Queueing analysis in healthcare. In: Patient flow: reducing delay in healthcare delivery. Springer, pp. 281-307. 
Greene, J., 2007. Emergency department flow and the boarded patient: How to get admitted patients upstairs. Annals of Emergency Medicine 49 (1), 68-70.

URL http://www.sciencedirect.com/science/article/pii/S0196064406025443

Gross, D., Harris, C. M., 1985. Fundamentals of queueing theory (2nd Ed.). John Wiley \& Sons, Inc., New York, NY, USA.

Guo, C.-H., Iannazzo, B., Meini, B., 2008. On the doubling algorithm for a (shifted) nonsymmetric algebraic Riccati equation. SIAM Journal on Matrix Analysis and Applications 29 (4), 1083-1100.

URL http://dx.doi.org/10.1137/060660837

Heragu, S. S., Srinivasan, M., 2011. Analysis of manufacturing systems via single-class, semi-open queuing networks. International Journal of Production Research 49 (2), 295-319.

URL http://dx.doi.org/10.1080/00207540903527378

Horváth, G., Van Houdt, B., 2012. A multi-layer fluid queue with boundary phase transitions and its application to the analysis of multi-type queues with general customer impatience. In: Proceedings of the 9th International Conference on Quantitative Evaluation of Systems (QEST). IEEE Computer Society Press, pp. 23-32.

Huang, J., Carmeli, B., Mandelbaum, A., 2015. Control of patient flow in emergency departments, or multiclass queues with deadlines and feedback. Operations Research 63 (4), 892-908.

URL http://dx.doi.org/10.1287/opre.2015.1389

Izady, N., Worthington, D., 2012. Setting staffing requirements for time dependent queueing networks: The case of accident and emergency departments. European Journal of Operational Research 219 (3), 531-540. URL http://www.sciencedirect.com/science/article/pii/S0377221711009805

Jia, J., Heragu, S. S., Mar. 2009. Solving semi-open queuing networks. Operations Research 57 (2), 391-401. URL http://dx.doi.org/10.1287/opre.1080.0627

Kelen, G. D., Kraus, C. K., McCarthy, M. L., Bass, E., Hsu, E. B., Li, G., Scheulen, J. J., Shahan, J. B., Brill, J. D., Green, G. B., 2006. Inpatient disposition classification for the creation of hospital surge capacity: A multiphase study. The Lancet 368 (9551), 1984-1990.

URL http://www.sciencedirect.com/science/article/pii/S0140673606698085

Kleinrock, L., 1975. Theory, Volume 1, Queueing Systems. Wiley-Interscience.

Kolb, E. M. W., Schoening, S., Peck, J., Lee, T., 2008. Reducing emergency department overcrowding: Five patient buffer concepts in comparison. In: Proceedings of the 40th Conference on Winter Simulation. WSC '08. Winter Simulation Conference, pp. 1516-1525.

URL http://dl.acm.org/citation.cfm?id=1516744.1517010

Lagershausen, S., 2012. Performance analysis of closed queueing networks. Springer.

Little, J. D. C., Graves, S. C., 2008. Building intuition: Insights from basic operations management models and principles. Springer US, Boston, MA, Ch. Little's Law, pp. 81-100.

URL http://dx.doi.org/10.1007/978-0-387-73699-0_5

Liu, Y., Whitt, W., 2012. Stabilizing customer abandonment in many-server queues with time-varying arrivals. Operations Research 60 (6), 1551-1564.

URL http://dx.doi.org/10.1287/opre.1120.1104

Lucas, R., Farley, H., Twanmoh, J., Urumov, A., Olsen, N., Evans, B., Kabiri, H., 2009. Emergency department patient flow: The influence of hospital census variables on emergency department length of stay. Academic Emergency Medicine 16 (7), 597-602.

URL http://dx.doi.org/10.1111/j.1553-2712.2009.00397.x 
Pines, J. M., Hilton, J. A., Weber, E. J., Alkemade, A. J., Al Shabanah, H., Anderson, P. D., Bernhard, M., Bertini, A., Gries, A., Ferrandiz, S., Kumar, V. A., Harjola, V.-P., Hogan, B., Madsen, B., Mason, S., Öhlén, G., Rainer, T., Rathlev, N., Revue, E., Richardson, D., Sattarian, M., Schull, M. J., 2011. International perspectives on emergency department crowding. Academic Emergency Medicine 18 (12), $1358-1370$.

URL http://dx.doi.org/10.1111/j.1553-2712.2011.01235.x

Ramaswami, V., 1999. Matrix analytic methods for stochastic fluid flows. In: D., S., P., H. (Eds.), Teletraffic Engineering in a Competitive World. Proceedings of the 16th International Teletraffic Congress, Elsevier Science B.V., Edinburgh,, pp. 1019 - 1030.

Ramaswami, V., Lucantoni, D. M., 1985. Stationary waiting time distribution in queues with phase type service and in quasi-birth-and-death processes. Communications in Statistics. Stochastic Models 1 (2), $125-136$.

Roy, D., 2016. Semi-open queuing networks: a review of stochastic models, solution methods and new research areas. International Journal of Production Research 54 (6), 1735-1752.

URL https://doi.org/10.1080/00207543.2015.1056316

Roy, D., Krishnamurthy, A., Heragu, S. S., Malmborg, C. J., 2012. Performance analysis and design trade-offs in warehouses with autonomous vehicle technology. IIE Transactions 44 (12), 1045-1060.

URL http://dx.doi.org/10.1080/0740817X.2012.665201

Saghafian, S., Austin, G., Traub, S. J., 2015. Operations research/management contributions to emergency department patient flow optimization: Review and research prospects. IIE Transactions on Healthcare Systems Engineering 5 (2), 101-123.

URL http://dx.doi.org/10.1080/19488300.2015.1017676

Saghafian, S., Hopp, W. J., Oyen, M. P. V., Desmond, J. S., Kronick, S. L., 2014. Complexity-augmented triage: A tool for improving patient safety and operational efficiency. Manufacturing \& Service Operations Management 16 (3), 329-345.

URL http://dx.doi.org/10.1287/msom.2014.0487

Sengupta, B., 1989. Markov processes whose steady state distribution is matrix-exponential with an application to the GI/PH/1 queue. Advances in Applied Probability 21 (1), 159-180.

URL http://www . jstor.org/stable/1427202

Sengupta, B., 1990. The semi-markovian queue: Theory and applications. Communications in Statistics. Stochastic Models 6 (3), 383-413.

URL http://dx.doi.org/10.1080/15326349908807154

Shi, P., Chou, M. C., Dai, J., Ding, D., Sim, J., 2015. Models and insights for hospital inpatient operations: Time-dependent ED boarding time. Management Science 62 (1), 1-28.

Shi, P., Dai, J., Ding, D., Ang, J., Chou, M., Xin, J., Sim, J., October 2013. Patient flow from emergency department to inpatient wards: Empirical observations from a Singaporean hospital. Tech. rep., Social Science Research Network.

Teow, K. L., El-Darzi, E., Foo, C., Jin, X., Sim, J., 2011. Intelligent analysis of acute bed overflow in a tertiary hospital in Singapore. Journal of Medical Systems 36 (3), 1873-1882.

URL http://dx.doi.org/10.1007/s10916-010-9646-1

Thompson, S., Nunez, M., Garfinkel, R., Dean, M. D., 2009. OR practice-efficient short-term allocation and reallocation of patients to floors of a hospital during demand surges. Operations Research 57 (2), 261-273.

Trzeciak, S., Rivers, E. P., 2003. Emergency department overcrowding in the United States: An emerging threat to patient safety and public health. Emergency Medicine Journal 20 (5), 402-405.

URL http://emj.bmj.com/content/20/5/402.abstract 
van der Linden, C., Lucas, C., van der Linden, N., Lindeboom, R., 2013. Evaluation of a flexible acute admission unit: Effects on transfers to other hospitals and patient throughput times. Journal of Emergency Nursing 39 (4), 340-345.

URL http://www.sciencedirect.com/science/article/pii/S0099176711005757

Viccellio, A., Santora, C., Singer, A. J., Thode Jr., H. C., Henry, M. C., 2009. The association between transfer of emergency department boarders to inpatient hallways and mortality: a 4-year experience. Annals of Emergency Medicine 54 (4), 487-491.

URL http://www.sciencedirect.com/science/article/pii/S0196064409002388

Viccellio, P., Zito, J. A., Sayage, V., Chohan, J., Garra, G., Santora, C., Singer, A. J., 2013. Patients overwhelmingly prefer inpatient boarding to emergency department boarding. The Journal of Emergency Medicine 45 (6), 942-946.

URL http://www.sciencedirect.com/science/article/pii/S0736467913008263

Villa-Roel, C., Guo, X., Holroyd, B. R., Innes, G., Wong, L., Ospina, M., Schull, M., Vandermeer, B., Bullard, M. J., Rowe, B. H., 2012. The role of full capacity protocols on mitigating overcrowding in EDs. The American Journal of Emergency Medicine 30 (3), 412-420. URL http://www.sciencedirect.com/science/article/pii/S0735675711000039

Wang, W., Wang, W., Li, R., 2012. Alternating-directional doubling algorithm for M-matrix algebraic Riccati equations. SIAM Journal on Matrix Analysis and Applications 33 (1), 170-194. URL http://dx.doi.org/10.1137/110835463

Wang, X., 2013. Emergency department staffing: A separated continuous linear programming approach. Mathematical Problems in Engineering 2013.

Wiler, J. L., Bolandifar, E., Griffey, R. T., Poirier, R. F., Olsen, T., 2013. An emergency department patient flow model based on queueing theory principles. Academic Emergency Medicine 20 (9), 939-946. URL http://dx.doi.org/10.1111/acem.12215

Yankovic, N., Green, L. V., 2011. Identifying good nursing levels: A queuing approach. Operations Research 59 (4), 942-955.

URL http://dx.doi.org/10.1287/opre.1110.0943

Yom-Tov, G. B., 2010. Queues in Hospitals: Queueing Networks with ReEntrant Customers in the QED Regime. Ph.D. thesis, Technion.

Yom-Tov, G. B., Mandelbaum, A., 2014. Erlang-R: A time-varying queue with Reentrant customers, in support of healthcare staffing. Manufacturing \& Service Operations Management 16 (2), 283-299.

URL http://dx.doi.org/10.1287/msom.2013.0474

\section{Appendix A. Calculating the steady-state distribution of the number of patients in the external queue}

To calculate the probability that there are $i$ patients in the external queue, we need to know both the probability that the FIL patient waiting time is $y$ hours and the probability that $i-1$ patients entered during that time window. For $\tau \geq 0$ :

$$
\begin{aligned}
& \operatorname{Pr}(X(\tau)=y)=\alpha \boldsymbol{p}_{-} \boldsymbol{T}_{-+}^{(\mathbf{0})} \exp (\boldsymbol{K} y) \mathbf{1}_{\overline{\boldsymbol{m}} \cdot} \\
& \operatorname{Pr}(\mathrm{i}-1 \text { arrivals in interval } y)=\frac{(\lambda y)^{i-1} \exp (-\lambda y)}{(i-1) !} .
\end{aligned}
$$


Combining these two equations yields:

$$
\begin{aligned}
\operatorname{Pr}\left(L_{e, q}=i\right) & =\int_{0}^{\infty} \operatorname{Pr}(X(\tau)=y) \operatorname{Pr}(\text { i-1 patients arrive in interval } y) d y \\
& =\int_{0}^{\infty} \alpha \boldsymbol{p}_{-} \boldsymbol{T}_{-+}^{(\mathbf{0})} \exp (\boldsymbol{K} y) \mathbf{1}_{\overline{\boldsymbol{m}}} \frac{(\lambda y)^{i-1} \exp (-\lambda y)}{(i-1) !} d y \\
& =\alpha \boldsymbol{p}_{-} \boldsymbol{T}_{-+}^{(\mathbf{0})} \int_{0}^{\infty} \exp (\boldsymbol{K} y) \frac{(\lambda y)^{i-1} \exp (-\lambda y)}{(i-1) !} d y \mathbf{1}_{\overline{\boldsymbol{m}}} \\
& =\alpha \boldsymbol{p}_{-} \boldsymbol{T}_{-+}^{(\mathbf{0})} \int_{0}^{\infty} \exp \left(\left(\boldsymbol{K}-\lambda \boldsymbol{I}_{\overline{\boldsymbol{m}}}\right) y\right) \frac{(\lambda y)^{i-1}}{(i-1) !} d y \mathbf{1}_{\overline{\boldsymbol{m}}} \\
& =\alpha \boldsymbol{p}_{-} \boldsymbol{T}_{-+}^{(\mathbf{0})} \lambda^{i-1}\left(\lambda \boldsymbol{I}_{\overline{\boldsymbol{m}}}-\boldsymbol{K}\right)^{-i} \mathbf{1}_{\overline{\mathbf{m}}}
\end{aligned}
$$

The last step in the derivation follows from:

$$
\begin{aligned}
& \int \exp \left(\left(\boldsymbol{K}-\lambda \boldsymbol{I}_{\overline{\boldsymbol{m}}}\right) y\right) \frac{(\lambda y)^{i-1}}{(i-1) !} d y \\
& =\frac{-\lambda^{i-1}\left(\lambda \boldsymbol{I}_{\overline{\boldsymbol{m}}}-\boldsymbol{K}\right)^{-i} \Gamma\left(i,\left(\lambda \boldsymbol{I}_{\overline{\boldsymbol{m}}}-\boldsymbol{K}\right) y\right)}{(i-1) !}+C .
\end{aligned}
$$

where $\Gamma\left(i,\left(\lambda \boldsymbol{I}_{\bar{m}}-\boldsymbol{K}\right) y\right)$ is the incomplete gamma function, and $C$ is an arbitrary constant. Because $i$ is a positive integer, $\Gamma\left(i,\left(\lambda \boldsymbol{I}_{\overline{\boldsymbol{m}}}-\boldsymbol{K}\right) y\right)=(i-1) ! \exp \left(\left(\boldsymbol{K}-\lambda \boldsymbol{I}_{\overline{\boldsymbol{m}}}\right) y\right) \sum_{k=0}^{i-1} \frac{\left(\left(\lambda \boldsymbol{I}_{\overline{\boldsymbol{m}}}-\boldsymbol{K}\right) y\right)^{k}}{k !}$. 


\section{Appendix B. Probability of starvation at the physician stage}

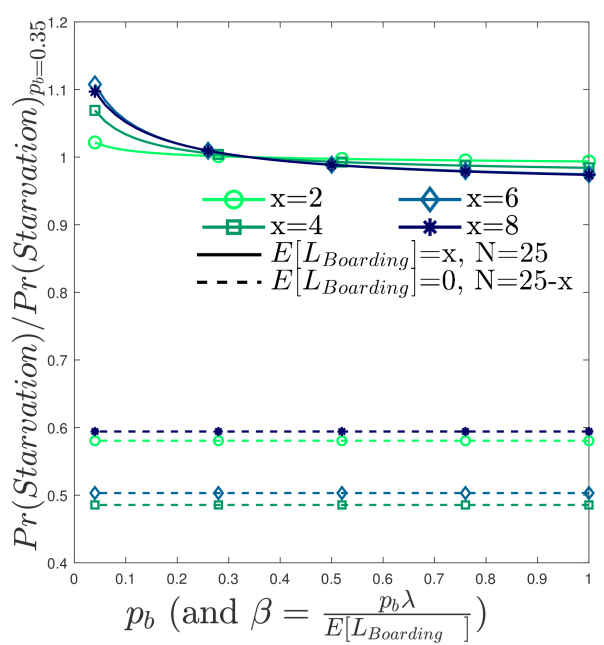

(a) Scenario HL

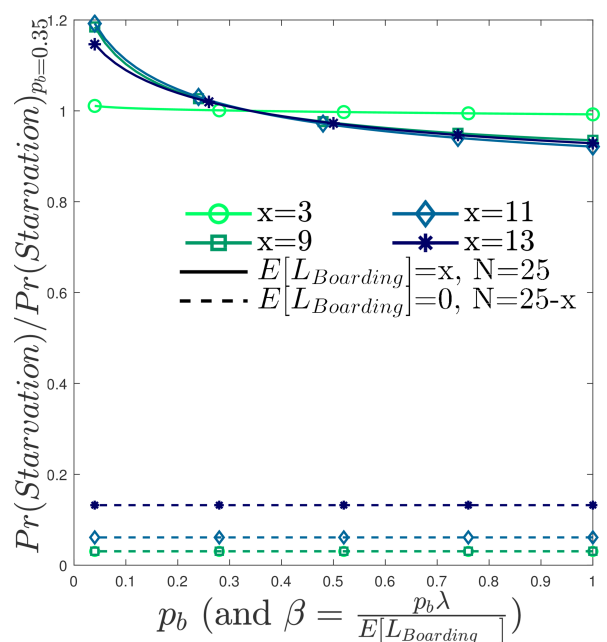

(b) Scenario LL

Figure B.15: Effect of changes in $\beta$ and $p_{b}$ on the probability that a physician is idle while there are patients in the external queue

\section{Appendix C. Probability density functions of the number of ED beds used for boarding}

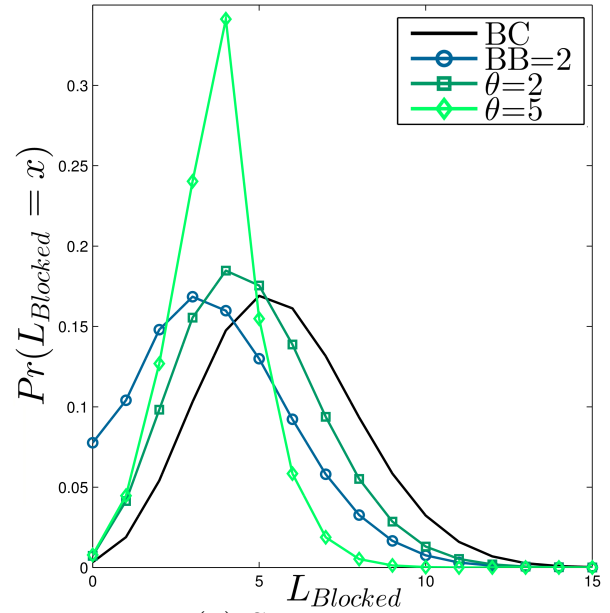

(a) Scenario HL



(b) Scenario LL

Figure C.16: Effect of a reactive or proactive policy on the probability density function of the number of ED beds used for boarding, compared with the base case (for $N=25$ ) 